CLINICAL STUDY

\title{
Circulating levels of GH predict mortality and complement prognostic scores in critically ill medical patients
}

\author{
Philipp Schuetz, Beat Müller ${ }^{1}$, Charly Nusbaumer ${ }^{2}$, Melanie Wieland ${ }^{2}$ and Mirjam Christ-Crain \\ Division of Endocrinology, Diabetes and Clinical Nutrition, Department of Internal Medicine, Diabetes and Clinical Nutrition, University Hospital Basel, \\ Basel, Switzerland, ${ }^{1}$ Department of Internal Medicine, Kantonsspital Aarau, Tellstrasse, CH-5001 Aarau, Switzerland and ${ }^{2}$ Department of Chemical \\ Pathology, University Hospital Basel, Basel, Switzerland \\ (Correspondence should be addressed to B Müller; Email: happy.mueller@unibas.ch)
}

\begin{abstract}
Background: Circulating levels of GH are increased during critical illness and correlate with outcome in children with meningococcal sepsis. We assessed the prognostic implications of GH on admission and during follow-up in critically ill adult patients admitted to a medical intensive care unit.

Materials and methods: We measured GH, IGF1 and IGF-binding protein3 (IGFBP-3) plasma concentrations in 103 consecutive critically ill patients and compared it with two clinical severity scores (APACHE II, SAPS II).

Results: Median GH levels on admission were similar in septic $(n=53)$ and non-septic $(n=50)$ patients and about 7 -fold increased in the 24 non-survivors as compared with survivors $(9.50$ (interquartile ranges (IQR) 3.53-18.40) vs 1.4 (IQR 0.63-5.04), P<0.0001). GH levels increased with increasing severity of sepsis (sepsis, severe sepsis, and septic shock, $P=0.019$ ). By contrast, IGF1 and IGFBP-3 did not correlate with severity of disease or mortality. Logistic regression models showed that $\mathrm{GH}$ and both clinical scores were independent predictors of mortality with a similar prognostic accuracy (GH: area under the curve (AUC) 0.81 (95\% confidence interval (CI), 0.71-0.92), APACHE II: AUC 0.71 (95\% CI, $0.58-0.83), P=0.16$, SAPS II: AUC 0.75 (95\% CI, 0.63-0.86, $P=0.36)$ ). GH improved the prognostic accuracy of the APACHE II score to an AUC of 0.78 (95\% CI, 0.66-090, $P=0.04)$ and tended to improve the SAPS II score to an AUC of 0.79 (95\% CI, 0.67-0.90, $P=0.09$ ).

Conclusion: GH plasma concentrations on admission are independent predictors for mortality in adult critically ill patients and may complement existing risk prediction scores, namely the APACHE II and the SAPS II score.
\end{abstract}

European Journal of Endocrinology 160 157-163

\section{Introduction}

Mortality in sepsis results from a potentially harmful host response to infection (1). Despite improved supportive care, the mortality of sepsis and septic shock still ranges between 25 and 70\% (2). During sepsis and critical illness, the activation of the anterior pituitary function is a major surrogate of the host's response to stress and leads to a continuum of neuroendocrine and metabolic changes $(3,4)$. This response per se is considered to be beneficial for patients, as it provides metabolic substrates and supports the integrity of host deference $(3,4)$. Activation of the hypothalamic-pituitary axis in critical illness leads to an alteration in the pulsatile release of $\mathrm{GH}$ from the somatotropes (3-5). During the early phases of critical illness, the pulse frequency augments leading to an increase in circulating levels of GH (6). Conversely, in the peripheral tissues there is an acquired peripheral $\mathrm{GH}$ resistance with down regulation of insulin-like growth factor 1 (IGF1) and GH binding proteins (IGFBP-3) leading to protein catabolism and a negative nitrogen balance (6). To convert the negative effects of peripheral $\mathrm{GH}$ deficiency, clinical trials with high dose $\mathrm{GH}$ treatment have been initiated. However, early termination was necessary because of an increased mortality observed in GH treated patients (7).

Enormous attempts have been undertaken to identify prognostic markers in the acute phase of critical illness because accurate risk prediction directs the diagnostic and therapeutic work up and may thus improve the mortality and morbidity of patients (8-12). In this context, endocrine markers including high serum cortisol and/or low tri-iodothyronine levels have been correlated with poor outcome in critically ill patients (3, $8,13,14)$. Interestingly, in children with meningococcal sepsis, non-surviving children had markedly higher GH levels and decreased IGF1 levels as compared with survivors (15). Another study in children with sepsis or septic shock found similar results (16). During prolonged critical illness, low serum concentrations of IGFBP-1 are a predictor for adverse outcome $(3,17)$ and a low 
IGF1/GH ratio identified patients with chronic heart failure at higher risk of cardiac death (18). Data about the prognostic value of $\mathrm{GH}$ in adult patients with acute critical illness or sepsis are lacking. Herein, we assessed the prognostic value of $\mathrm{GH}$ concentrations on admission and during follow up in critically ill patients in a medical intensive care unit (ICU).

\section{Materials and methods}

\section{Patients}

This is a prospective study evaluating GH, IGF1 and IGFBP-3 concentrations in plasma samples of 103 consecutive critically ill patients admitted to the medical ICU of the University Hospital of Basel, Switzerland. The primary endpoint of this study was the assessment of the prognostic value of endocrine dysfunctions in critically ill patients ('PEDCRIP'-study) (19).

Over a 9-month period 103 consecutive critically ill patients admitted to the medical ICU were included. Two patients were included twice into the study after an interval of 4 and 7-month and with different admission diagnoses. All patients were followed until hospital discharge or death respectively. Vital signs, clinical status, and severity of disease were assessed daily and the commonly used physiological APACHE II score and the SAPS II score were calculated on the basis of the patients unique set of parameters as described elsewhere $(11,12,20)$. When feasible, consent was obtained prior to enrolment in conscious patients, otherwise the consent was obtained from the patients' next of kin. The study protocol had prior approval by the local ethical review board Ethikkommisson beider Basel.

Patients were classified into having sepsis including severe sepsis and septic shock or a systemic inflammatory response syndrome (SIRS) without evidence of infection using international, standardized criteria $(21,22)$. In brief, SIRS was characterized by the presence of at least two of the following four clinical criteria: fever or hypothermia (temperature $>38{ }^{\circ} \mathrm{C}$ or $<36{ }^{\circ} \mathrm{C}$ ); tachycardia ( $>90$ beats $/ \mathrm{min}$ ); tachypnoea ( $>20 \mathrm{breaths} / \mathrm{min}$ or $<32 \mathrm{mmHg}$ or the need for mechanical ventilation support); and an altered white blood cell (WBC) count $(>12000$ cells $/ \mu$ or $<4000$ cells $/ \mu \mathrm{l})$ or the presence of $>10 \%$ band forms. Sepsis was defined as SIRS with an infection. In case of uncertainty of infection, a complete, retrospective patient chart review including results of microbiological cultures, chest radiographs, and when available, post-mortem examination reports was performed by an infectious disease specialist. An isolated microorganism was considered to be pathogenic if recovered within a 24-hour-period before or after the onset of the systemic response. Microbiological tests and antibiotic therapy were prescribed by physicians on duty according to common practice, without interference by the research team.

\section{Assays}

Results of the routine blood analyses including WBC $\left(\times 10^{9}\right)$, C-reactive protein $(\mathrm{CRP} ; \mathrm{mg} / \mathrm{ml})$ and lactate $(\mathrm{mmol} / \mathrm{l})$ were recorded in all patients. Plasma on admission, on the morning of the 2-day inclusion and before discharge from the ICU or before death respectively, was collected at the time of blood sampling in plastic tubes containing EDTA. They were placed on ice, centrifuged at $3000 \boldsymbol{g}$ and plasma was frozen at $-70{ }^{\circ} \mathrm{C}$ until assayed. Serum $\mathrm{GH}(\mathrm{ng} / \mathrm{ml})$ was measured by an immunoradiometric assay (CIS-Bio International, Gif-sur-Yvette, France) with a functional detection limit of $0.08 \mathrm{ng} / \mathrm{ml}$. Concentrations of total IGF1 (nmol/l) and IGFBP-3 (nmol/l) were determined with radioimmuno assays (Utrecht Medical Center, Utrecht, The Netherlands). CRP (mg/l) was measured by an enzyme immunoassay (EMIT CRP assay; Merck Diagnostica) and a value of $>10 \mathrm{mg} / \mathrm{l}$ was considered to be abnormally elevated. Lactate concentrations ( $\mathrm{mmol} / \mathrm{l}$ ) were measured enzymatically (Du Pont, Wilmington, DE, USA; reference range $1.1-2.0 \mathrm{mmol} / \mathrm{l})$. Procalcitonin (PCT; ng/ml) was measured using the LUMItest assay (Brahms AG, Hennigsdorf, Germany). Serum interleukin-6 (IL-6) concentrations (ng/ml) were measured with a commercially available quantitative sandwich enzyme immunoassay (CLB; Pelikine Compact, Amsterdam, Netherlands) with a limit of detection of $0.6 \mathrm{pg} / \mathrm{ml}$.

\section{Statistical analysis}

Logarithmic transformation was performed to achieve a normal distribution for skewed variables (CRP PCT, GH and (IL-6)). Discrete variables are expressed as counts (percentage) and continuous variables as medians and interquartile ranges (IQR) unless stated otherwise. Frequency comparison was done by $\chi^{2}$-test. For group comparisons the Mann-Whitney $U$ test was used if only two groups were compared and the Kruskal-Wallis oneway analysis of variance was used if more than two groups were being compared. To compare the prognostic accuracy of different prognostic parameters, odds ratios were estimated using univariate and multivariate regression models and receiver-operating characteristic (ROC) curves were calculated with the AUC as an overall prognostic measure. Thereby, outcomes were either death or survival until hospital discharge. To estimate the potential clinical relevance of GH measurements, we used likelihood-ratio tests to determine whether logistic regression models that included measurements of $\mathrm{GH}$ and either the APACHE II score or the SAPS II score provided a significant better fit than did logistic regression models limited to the clinical scores alone. Correlation analyses were performed by 
using Spearman rank correlation. All testing was twotailed and $P$-values less than 0.05 were considered to indicate statistical significance. All calculations were performed using STATA 9.2 (Stata Corp., College Station, TX, USA).

\section{Results}

\section{Descriptive characteristics of the patients}

The median age of the 103 patients included in this study was 57 years (IQR 46-69) and $45.6 \%$ were females $(n=47)$. In 50 patients $(48.5 \%)$, a SIRS without evidence of underlying infection was found. The principal diagnoses in these patients included neurological disease (e.g. stroke, $n=16$ ), respiratory failure $(n=13)$, cardiovascular disease (e.g. myocardial infarction and cardiogenic shock, $n=12)$, gastrointestinal disease (e.g. gastric bleeding, $n=6$ ), trauma $(n=2)$, and neoplastic disease $(n=1)$. Sepsis was diagnosed in 53 patients including severe sepsis $(n=15)$ and septic shock $(n=16)$. The principal site of infection in septic patients was the lung $(n=37)$, the gastrointestinal tract $(n=5)$, the urogenital tract $(n=4)$, and primary blood stream infection $(n=2)$. Blood cultures revealed growth of typical microorganisms in 31 patients including Streptococcus pneumoniae $(n=9)$, Escherichia coli $(n=6)$, Pseudomonas aeruginosa $(n=6)$, Enterobacteria $(n=2)$, Haemophilus influenzae $(n=2)$, and Staphylococcus aureus $(n=1)$. The need for blood pressure support with vasoactiva on admission was $30.0 \%$ in patients with SIRS and $62.2 \%$ in patients with sepsis. The median length of stay in the medical ICU was 4.1 days (IQR 2.08.7). Risk assessment on admission to the ICU revealed a median APACHE II score of 21 points (IQR 17-28) and a median SAPS score of 54 (43-66). The in-hospital mortality rate of all patients was $23.3 \%$ after a median of 3.6 days (IQR 1.6-6.4). From the 53 septic patients 13 died $(24.5 \%)$, while 11 out of the 50 SIRS patients $(23.4 \%)$ did not survive the hospital stay. Additional baseline data of the patients are shown in Table 1 .

\section{GH, IGF1 and IGFBP-3 levels on admission and follow up}

Median GH levels on admission to the ICU were increased in all patients (2.42 (IQR 0.85-6.96)) and were similar in patients with and without sepsis $(P=0.77)$. In septic patients, there was an increase in $\mathrm{GH}$ levels with increasing severity of sepsis (i.e. sepsis, severe sepsis, septic shock; ANOVA $P=0.019$; Fig. 1). In addition, high-risk patients in the highest quartile of the APACHE II score and in the highest quartile of the SAPS II score had significantly higher GH concentrations on admission as compared with patients in the lower quartiles $(P<0.01$ and 0.005; Fig. 2). Median follow up measurements of $\mathrm{GH}$ in all patients after $24 \mathrm{~h}$
Table 1 Baseline characteristics of the 103 critically ill patients.

\begin{tabular}{ll}
\hline Demographic characteristics & \\
Age - years & $59(46-68)$ \\
Male sex & $56(54.4 \%)$ \\
Weight $(\mathrm{kg})$ & $70.1(62.5-81.3)$ \\
BMI $\left(\mathrm{kg} / \mathrm{m}^{2}\right)$ & $24.2(22.1-27.3)$ \\
Clinical findings & \\
Heart rate $(\mathrm{bpm})$ & $107(82-125)$ \\
Systolic blood pressure $(\mathrm{mmHg})$ & $105(92-128)$ \\
Respiratory rate & $16(12-25)$ \\
Temperature $\left({ }^{\circ} \mathrm{C}\right)$ & $36.7(35.8-37.6)$ \\
Laboratory findings & \\
C-reactive protein $(\mathrm{mg} / \mathrm{l})$ & $81(17-200)$ \\
Procalcitonin ( $\mathrm{\mu g} / \mathrm{l})$ & $1.1(0.3-4.8)$ \\
White blood cells $\left(\times 10^{9}\right)$ & $11.1(8.2-15.2)$ \\
GH (ng/ml) & $2.42(0.85-6.96)$ \\
IGF-I (nmol/l) & $13.9(8.7-18.2)$ \\
IGFBP-3 (nmol/l) & $1.7(1.3-2.8)$ \\
Lactate $(\mathrm{mmol} / \mathrm{l})$ & $1.85(1.3-3.5)$ \\
IL-6; $(\mathrm{ng} / \mathrm{ml})$ & $69.4(12.5-284)$ \\
Risk assessment & \\
APACHE II (points) & $21(17-28)$ \\
SAPS II score II (points) & $54(43-66)$ \\
Outcome & \\
Mortality number (\%) & $24(23.3 \%)$
\end{tabular}

BMI, body mass index; MAP, middle arterial blood pressure; IGF-I, insulinlike growth factor-1; IGFBP-3, IGF-binding protein-3; if not stated otherwise median values and the interquartile range (in brackets) is given.

(1.56 (IQR 0.70-5.26), $P=0.42$ ) and at discharge from the ICU (2.04 (IQR 0.85-6.19), P=0.62) were not significantly different from the initial measurements.

Patients with sepsis had significantly lower IGF1 levels (10.6 (IQR 7.6-15.6) vs 15.2 (IQR 12.2-21.5), $P=0.002)$ and lower IGFBP-3 levels (1.5 (IQR 1.1-2.3) vs $2.0(1.5-3.0), P=0.001)$ as compared with patients with SIRS. Neither IGF1 nor IGFBP-3 levels correlated with severity of sepsis and both were similar in patients in the highest APACHE II quartile $(P=0.3$ and $P=0.25)$ and in the highest SAPS II quartile $(P=0.27$ and $P=0.15)$. Because this study focuses on risk prediction in the acute situation, IGF1 and IGFBP-3 levels were only measured on admission and not reassessed during follow up.

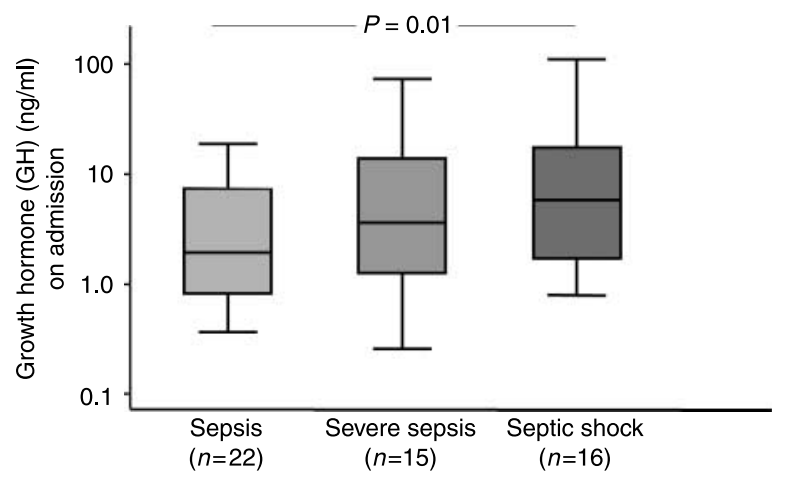

Figure $1 \mathrm{GH}$ concentrations significantly increase with increasing severity of sepsis defined as severe sepsis $(n=15)$ and septic shock $(n=16)$. 


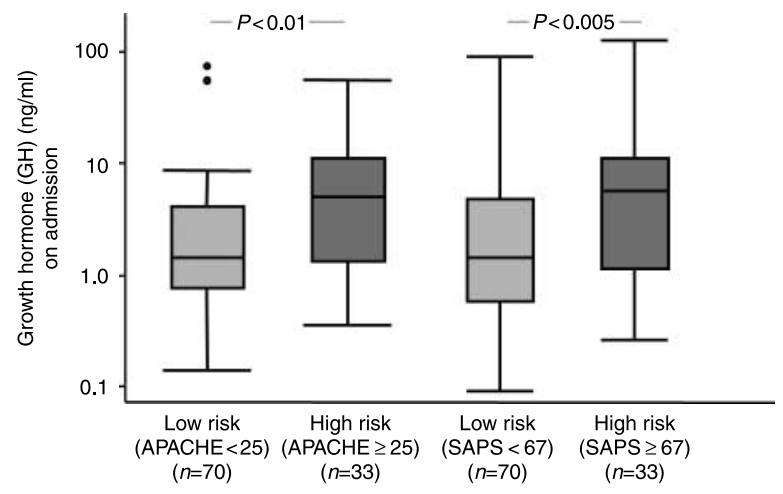

Figure $2 \mathrm{GH}$ concentrations are significantly higher in high risk patients in the highest quartile of the APACHE ( $\geq 25$ points) and the SAPS II ( $\geq 67$ points) score.

\section{GH, IGF1 and IGFBP-3 levels in survivors and non-survivors}

Median GH levels on admission were about 7-fold increased in the 24 patients who subsequently died as compared with the survivors (9.50 (IQR 3.53-18.40) vs 1.4 (IQR 0.63-5.04), $P<0.0001$ ). This increase was less pronounced for the APACHE II score (27 (IQR 1933 ) vs 21 (IQR 16-26), $P=0.005$ ) and the SAPS II score (67 (IQR 53-83) vs 50 (IQR 38-62), $P=0.003$ ). By contrast, WBC (10.2 (IQR 8.2-13.5) vs 13.6 (IQR 8.2-18.6), $P=0.1$ ), CRP (84 (IQR 36-260) vs 74 (IQR 14-194), $P=0.48$ ) and PCT (2.4 (IQR 0.5-12.3) vs 1.1 (IQR 0.3-4.4), $P=0.13$ ) levels were similar in survivors and non-survivors. In addition, metabolic parameters on admission including glucose levels (mmol/l; 7.5 (IQR 6.0-10.2) vs 9.0 (IQR 6.7-11.7), $P=0.38$ ) and albumin levels (g/l; 25 (IQR 21-32) vs 24 (IQR 19$32), P=0.21$ ), the body mass index (24.2 (IQR 22.1$27.8)$ vs 24.4 (IQR 23.4-25.7), $P=0.97$ ) and the age of patients (56 (IQR 46-67) vs 63 (IQR 52-74) $P=0.13)$ were similar in survivors and non-survivors. As demonstrated in Fig. 3, the difference in $\mathrm{GH}$

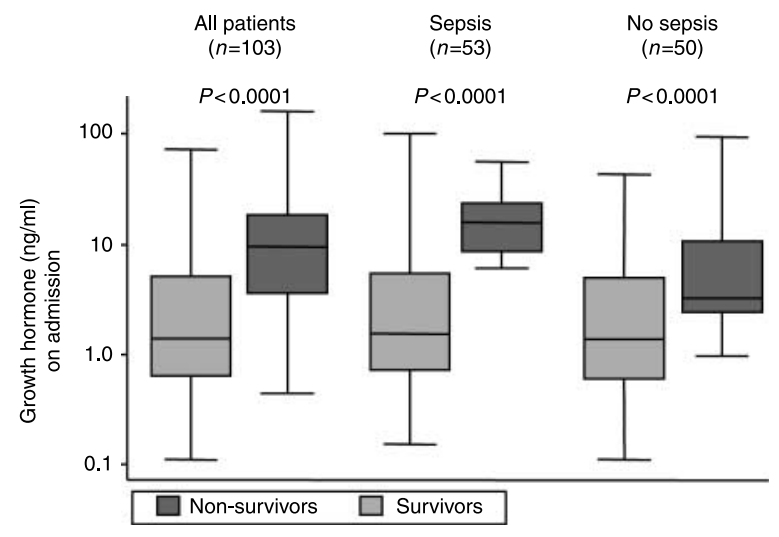

Figure $3 \mathrm{GH}$ concentrations in survivors and non-survivors. $\mathrm{GH}$ concentrations are increased in non-survivors overall, and in subgroups of patients with sepsis and patients with SIRS. concentration between survivors and non-survivors was independently observed in all patients, and in subgroups of septic and SIRS patients. The increase of $\mathrm{GH}$ in non-survivors was found on admission to the ICU and during follow-up of patients (Fig. 4). By contrast, IGF1 and IGFBP-3 levels on admission in survivors and non-survivors were similar (14 (IQR 9.8-17.8) vs 12.4 (7.1-19.7), $P=0.34$ and 1.8 (IQR $1.3-2.8$ ) vs 1.7 (IQR $1.1-2.4), P=0.36)$.

To compare the prognostic value of GH with IGF1, IGFBP-3, and other well known predictors of adverse outcome including age of patients, the APACHE II score, the SAPS II score, glucose levels, WBC, CRP, PCT, lactate, IL-6, and evidence of underlying sepsis to predict in-hospital mortality, we calculated a univariate logistic regression model. As illustrated in Table 2, GH, IL-6, lactate, the APACHE II score, and the SAPS II score were significant predictors of in-hospital mortality, in contrast to all other predictors. When entered in a multivariate regression model with either the APACHE II score or the SAPS II score, only GH $(P=0.001$ and $P=0.008)$, but not IL-6 $(P=0.58$ and $P=0.86)$ and lactate $(P=0.08$ and $P=0.2)$ was an independent predictor for death. In addition, when adding glucose levels on admission in the multivariate model, GH remained an independent predictor for outcome (data not shown).

To assess the prognostic accuracy of GH as compared with the APACHE II score, the SAPS II score, CRP, WBC, PCT, lactate, and IL- 6 to predict mortality, ROC curves were calculated. The discriminatory ability of GH (AUC 0.81 (95\% confidence interval (CI) $0.71-0.92)$ ) was within the range of the APACHE II score (AUC 0.71 (95\% CI $0.58-0.83), P=0.16$ ) and the SAPS II score (AUC $0.76(0.64-0.88), P=0.35)$ and significantly better than all other parameters (Table 3). A combined model including GH and the APACHE II score (AUC 0.78 (95\% CI 0.66.090) improved the prognostic accuracy of the APACHE II score alone $(P=0.04)$. Similarly, GH tended to improve the prognostic accuracy of the SAPS

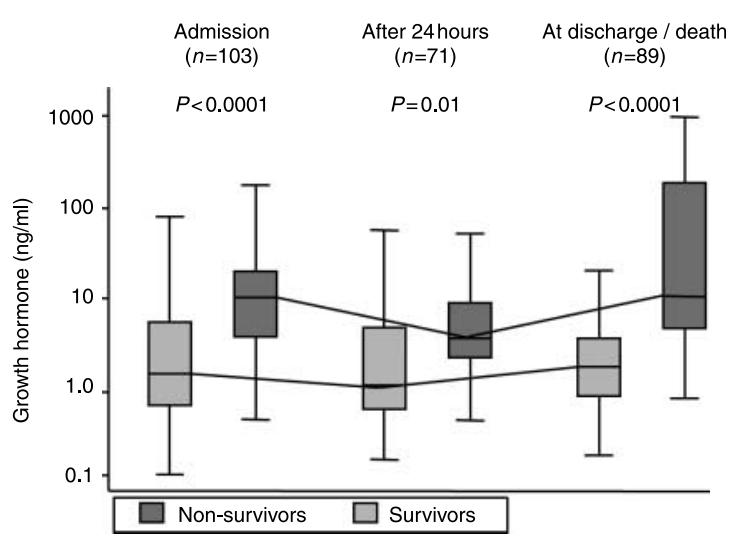

Figure $4 \mathrm{GH}$ concentrations are increased in non-survivors on admission and during follow up. 
Table 2 Prediction of mortality in univariate logistic regression.

\begin{tabular}{|c|c|c|}
\hline Predictor & Odds ratio $(95 \% \mathrm{Cl})$ & $P$ value \\
\hline $\mathrm{GH}^{\mathrm{a}}$ & $8.2(2.29-23.1)$ & $<0.0001$ \\
\hline IGF-I & $0.99(0.94-1.05)$ & 0.88 \\
\hline IGFBP-3 & $0.81(0.48-1.34)$ & 0.42 \\
\hline Age & $1.02(0.99-1.05)$ & 0.19 \\
\hline Glucose levels & $1.07(0.95-1.19)$ & 0.25 \\
\hline White blood cells & $1.07(0.99-1.16)$ & 0.075 \\
\hline C-reactive protein ${ }^{a}$ & $1.32(0.62-2.79)$ & 0.46 \\
\hline Procalcitonin $^{\mathrm{a}}$ & $1.61(0.94-2.78)$ & 0.08 \\
\hline Lactate & $1.36(1.07-1.73)$ & 0.013 \\
\hline IL-6 $6^{\mathrm{a}}$ & $2.42(1.36-4.31)$ & 0.003 \\
\hline APACHE II & $1.12(1.04-1.20)$ & $<0.0001$ \\
\hline SAPS II & $1.07(1.03-1.10)$ & $<0.0001$ \\
\hline Underlying sepsis & $1.15(0.46-2.88)$ & 0.76 \\
\hline
\end{tabular}

CI, confidence interval; IGF-I, Insulin-like growth factor-I; IGFBP-3 IGFbinding protein-3.

aLogarithmic transformation was performed to achieve a normal distribution for skewed variables.

II score in a combined model (AUC 0.79 (95\% CI 0.67-0.90), $P=0.09$ ).

To assess whether or not the higher GH level and the lower IGF1 level were worse for the patient or whether or not it is the high-GH levels alone that are worse for the patient, we calculated a GH/IGF1 ratio. The prognostic information of this ratio (AUC $0.78(95 \%$ CI $0.68-0.89$ ), $P=0.27$ ) was in the range of single $\mathrm{GH}$ measurements suggesting that the absolute increase in GH levels rather than a decrease in IGF1 levels correlates with outcome.

At the optimal GH cut-off of $6.5 \mathrm{ng} / \mathrm{ml}$ on admission, the sensitivity was $70 \%$ and the specificity was $85 \%$ to predict mortality with positive and negative predictive values of 57 and $91 \%$. The respective sensitivities, specificities, and positive and negative predictive values at a cut-off of $1.0 \mathrm{ng} / \mathrm{ml}$ were $96,38,31$ and 97,48 , 93, 65 and $86 \%$ for a cut-off of $10.0 \mathrm{ng} / \mathrm{ml}$ respectively.

Because two patients were included twice in the analysis, we performed a sensitivity analysis after exclusion of these two patients. The results of the sensitivity analysis were not different to the result of the

Table 3 Receiver operating curves for the prediction of mortality $(n=24)$ in all 103 critically ill patients on admission.

\begin{tabular}{lll}
\hline Parameter & AUC $(95 \% \mathrm{Cl})$ & $P$ \\
\hline GH & $0.81(0.71-0.92)$ & \\
White blood cells & $0.62(0.47-0.77)$ & 0.007 \\
C-reactive protein & $0.55(0.40-0.70)$ & 0.003 \\
Procalcitonin & $0.6(0.46-0.74)$ & 0.02 \\
Lactate & $0.66(0.53-0.80)$ & 0.04 \\
IL-6 & $0.71(0.59-0.83)$ & 0.05 \\
APACHE II & $0.71(0.58-0.83)$ & 0.16 \\
Combined model & $0.78(0.66 .090)$ & 0.59 \\
$\quad$ (APACHE II/GH) & & \\
SAPS II & $0.76(0.64-0.88)$ & 0.35 \\
Combined model (SAPS & $0.79(0.67-0.90)$ & 0.36 \\
$\quad$ II/GH) & & \\
\hline
\end{tabular}

AUC, area under the ROC curve; $\mathrm{Cl}$, confidence interval. whole study cohort in regard to logistic regression analysis and the prognostic accuracy in ROC curves.

\section{Discussion}

In this study, plasma GH levels on admission and during follow-up were increased in unselected septic and nonseptic critically ill patients from a medical ICU. GH levels correlated with severity of disease and were about 7-fold increased in patients who subsequently died. Importantly, GH was an independent predictor for mortality and improved the discriminatory ability of the APACHE II and the SAPS II score to identify those patients at high risk of dying in both septic and non-septic patients.

Studies on circulating GH levels in critically ill patients in the ICU setting have largely been conducted in the $70 \mathrm{~s}$ and $80 \mathrm{~s}$ of the past century $(3,6,23,24)$. These studies focused primarily on the pathophysiology of the pituitary alterations during critical illness. From these early studies, the severity-dependent increase of GH in patients with adverse medical outcomes is well documented and thus per se not a new finding. The finding that GH measurement improves the discriminatory ability of the APACHE II and the SAPS II scores, however, extends these early findings as the clinical value of GH has never been interpreted in the context of risk stratification of patients. Accordingly, none of the prognostic scores used in critically ill patients includes $\mathrm{GH}$ measurements, despite the promising early results. In this study, we measured only single GH concentrations at various time points of patients on admission to the ICU. GH is secreted in a pulsatile fashion and shows large variations during the day, although during severe infective illness elevated mean plasma GH levels without low trough values between GH peaks have been reported (15). A standardized GH profile may even show a higher prognostic accuracy, but would not be applicable in clinical practice $(15,16)$.

Two main mechanisms might explain the poor prognosis of patients with high GH concentrations. First, GH increases upon the individual stress level of patients and thus mirrors the stress associated with the severity and extent of illness $(3,4,24)$. Second, there is considerable evidence that $\mathrm{GH}$ resistance and the resulting increase in $\mathrm{GH}$ concentrations per se have deleterious effects in critically ill patients (7). Trials with recombinant $\mathrm{GH}$ have been conducted aiming at overcoming GH resistance and preventing the complications of GH resistance induced catabolism, until a multinational study revealed that critically ill patients treated with GH had an increase in mortality due to uncontrolled infections and development of multiple organ failure (7). Different pathophysiological mechanisms have been put forward to explain the excess in mortality observed upon $\mathrm{GH}$ treatment including modulation of the immune system, production of reactive oxygen species and proinflammatory 
cytokines, and an increase in susceptibility to endotoxins $(7,25,26)$. In addition, it has been postulated that $\mathrm{GH}$ stimulates lipolysis, interferes with thyroid and adrenocortical function and prevents the mobilization of glutamine from the muscles leaving less glutamine available for hepatic glutathione production and for rapidly dividing cells, namely leukocytes (7, 27-29).

The pulsatile secretion of GH shows an increased basal level with an unchanged frequency and amplitude of the secretion pulses in critically ill and septic patients (30). Despite this increased stimulation by GH the levels of IGF1 and its binding protein (IGFBP-3) are low, indicating hepatic GH resistance. This anabolic resistance is thought to contribute to the prolonged whole body protein breakdown with increased susceptibility for infections and thus delayed recovery. Some studies suggested that low concentrations of IGF1 and IGFBP-3 predict outcome in prolonged critical illness $(3,31,32)$. In this study, we found decreased IGF1 and IGFBP-3 levels in all critically ill patients, especially in septic patients. However, neither IGF1 nor IGFBP-3 correlated with severity of illness and levels were similar in survivors and non-survivors.

In the assessment and management of critically ill patients, knowledge of prognostic factors is crucial to estimate the risk for mortality. For this purpose, the APACHE II and the SAPS II scores are extensively validated prognostic assessment tools based on routine physiologic measurements, age, and previous health status $(11,12)$. With AUCs of 0.71 and 0.76 as found in this study, the prognostic accuracy of the clinical scores still has room for improvement. In this context, there is interest for new readily measurable biomarkers mirroring distinct pathogenetic mechanisms that could independently ameliorate existing risk scores. The utility of a biomarker is defined by the degree it improves clinical decision making and adds timely information beyond that of readily available information from clinical examination (10). The information of a biomarker may provide new insights into the pathophysiology and prognosis of the disease process thereby facilitating risk stratification and monitoring of therapy as a surrogate outcome measure. Our study demonstrates that GH measurement has a high prognostic utility as an early and independent risk predictor for death with the potential to complement and thereby improve the APACHE II and SAPS II scores.

Some limitations should be considered in interpreting our results. First, this is an observational study and the clinical benefits of GH measurements must be validated in a prospective design with clinical outcomes. Secondly, $\mathrm{GH}$ is influenced by different metabolic and other factors and we did not in detail assess the influence of how glucose control, insulin administration, and nutrition on the course of GH levels. Nevertheless, GH levels on admission were measured in treatment-naive acutely-ill patients transferred to the ICU and GH remained an important independent outcome predictor when adding initial glucose levels in multivariate regression models. In addition, age and BMI of patients, and metabolic factors including albumin levels and glucose levels were similar in survivors and non-survivors. Thirdly, we measured total $\mathrm{GH}$ levels and not the biologically active free $\mathrm{GH}$ concentrations that may show discordant results and a potentially even better prognostic accuracy. Similarly, a GH profile instead of only single measurements of this pulsatile secreted hormone may show better results. Forthly, the number of events (i.e. deaths) in this analysis is of only moderate size limiting the statistical power. Fifthly, in addition to the changes in the GH/IGF1 axis and the IGFBP-3 levels, previous studies reported a significant elevation in the level of IGFBP-1 in critical illness and a negative correlation between high levels of IGFBP-1 and survival (15). Unfortunately, we did not evaluate IGFBP levels in this study. For these reasons, our results are rather hypothesis-generating than definite.

In conclusion, in this study, GH had a high predictive capacity in adult critically ill patients with and without sepsis. If validated in future studies, GH may complement established risk scores for prognostication and stratification of critically ill medical patients.

\section{Declaration of interest}

All authors declare that they have no conflict of interest.

\section{Funding}

This research did not receive any specific grant from any funding agency in the public, commercial or not-for-profit sector.

\section{Acknowledgements}

The authors wish to thank the laboratory of chemical pathology of the University Hospital Basel, particularly Fausta Chiaverio, Peter Huber, and the staff of the medical ICU, namely, Rudolf Ritz and Hartmut Schächinger for their support during the study. We thank Andrea Ernst for critical discussions of the manuscript.

\section{References}

1 Cohen J. The immunopathogenesis of sepsis. Nature $2002 \mathbf{4 2 0}$ 885-891.

2 Russell JA. Management of sepsis. New England Journal of Medicine 2006355 1699-1713.

3 Van den Berghe G. The neuroendocrine response to stress is a dynamic process. Best Practice and Research. Clinical Endocrinology and Metabolism 200115 405-419.

4 Vanhorebeek I \& Van den Berghe G. The neuroendocrine response to critical illness is a dynamic process. Critical Care Clinics 200622 $1-15$.

5 Van den Berghe G. Dynamic neuroendocrine responses to critical illness. Frontiers in Neuroendocrinology 200223 370-391.

6 Ross R, Miell J, Freeman E, Jones J, Matthews D, Preece M \& Buchanan C. Critically ill patients have high basal growth hormone levels with attenuated oscillatory activity associated with low levels of insulin-like growth factor-I. Clinical Endocrinology 199135 47-54. 
7 Takala J, Ruokonen E, Webster NR, Nielsen MS, Zandstra DF, Vundelinckx G \& Hinds CJ. Increased mortality associated with growth hormone treatment in critically ill adults. New England Journal of Medicine 1999341 785-792.

8 Christ-Crain M, Stolz D, Jutla S, Couppis O, Muller C, Bingisser R, Schuetz P, Tamm M, Edwards R, Muller B \& Grossman AB. Free and total cortisol levels as predictors of severity and outcome in community-acquired pneumonia. American Journal of Respiratory and Critical Care Medicine 2007176 913-920.

9 Christ-Crain M, Morgenthaler NG, Struck J, Harbarth S, Bergmann A \& Muller B. Mid-regional pro-adrenomedullin as a prognostic marker in sepsis: an observational study. Critical Care 20059 R816-R824.

10 Marshall JC. Biomarkers of sepsis. Current Infectious Disease Reports 20068 351-357.

11 Knaus WA, Draper EA, Wagner DP \& Zimmerman JE. APACHEII: a severity of disease classification system. Critical Care Medicine 1985 13 818-829.

12 Knaus WA, Zimmerman JE, Wagner DP, Draper EA \& Lawrence DE. APACHE - acute physiology and chronic health evaluation: a physiologically based classification system. Critical Care Medicine 19819 591-597.

13 Gangemi EN, Garino F, Berchialla P, Martinese M, Arecco F, Orlandi F \& Stella M. Low triiodothyronine serum levels as a predictor of poor prognosis in burn patients. Burns $200834817-824$.

14 Leon-Sanz M, Lorente JA, Larrodera L, Ros P, Alvarez J, Esteban AE \& Landin L. Pituitary-thyroid function in patients with septic shock and its relation with outcome. European Journal of Medical Research 19972 477-482.

15 de Groof F, Joosten KF, Janssen JA, de Kleijn ED, Hazelzet JA, Hop WC, Uitterlinden P, van Doorn J \& Hokken-Koelega AC. Acute stress response in children with meningococcal sepsis: important differences in the growth hormone/insulin-like growth factor I axis between nonsurvivors and survivors. Journal of Clinical Endocrinology and Metabolism 200287 3118-3124.

16 Onenli-Mungan N, Yildizdas D, Yapicioglu H, Topaloglu AK, Yuksel B \& Ozer G. Growth hormone and insulin-like growth factor 1 levels and their relation to survival in children with bacterial sepsis and septic shock. Journal of Paediatrics and Child Health $200440221-226$.

17 Van den Berghe G, de Zegher F \& Bouillon R. Clinical review 95: acute and prolonged critical illness as different neuroendocrine paradigms. Journal of Clinical Endocrinology and Metabolism 1998 83 1827-1834.

18 Petretta M, Colao A, Sardu C, Scopacasa F, Marzullo P, Pivonello R, Fontanella L, de Caterina M, de Simone A \& Bonaduce D. NT-proBNP, IGF-I and survival in patients with chronic heart failure. Growth Hormone and IGF Research $200717288-296$.

19 Muller B, Becker KL, Schachinger H, Rickenbacher PR, Huber PR, Zimmerli W \& Ritz R. Calcitonin precursors are reliable markers of sepsis in a medical intensive care unit. Critical Care Medicine 2000 28 977-983.

20 Le Gall JR, Lemeshow S \& Saulnier F. A new simplified acute physiology score (SAPS II) based on a European/North American multicenter study. Journal of the American Medical Association 1993 270 2957-2963.

21 Bone RC, Balk RA, Cerra FB, Dellinger RP, Fein AM, Knaus WA, Schein RM \& Sibbald WJ. Definitions for sepsis and organ failure and guidelines for the use of innovative therapies in sepsis. The ACCP/SCCM Consensus Conference Committee. American College of Chest Physicians/Society of Critical Care Medicine. Chest 1992 101 1644-1655.

22 American College of Chest Physicians/Society of Critical Care Medicine Consensus Conference: definitions for sepsis and organ failure and guidelines for the use of innovative therapies in sepsis Critical Care Medicine 199220 864-874

23 Van den Berghe G, de Zegher F, Veldhuis JD, Wouters P, Awouters M, Verbruggen W, Schetz M, Verwaest C, Lauwers P, Bouillon R \& Bowers CY. The somatotropic axis in critical illness: effect of continuous growth hormone (GH)-releasing hormone and GH-releasing peptide-2 infusion. Journal of Clinical Endocrinology and Metabolism 199782 590-599.

24 Van den Berghe G. Endocrine changes in critically ill patients. Growth Hormone and IGF Research 19999 77-81.

25 Warwick-Davies J, Lowrie DB \& Cole PJ. Growth hormone is a human macrophage activating factor. Priming of human monocytes for enhanced release of H2O2. Journal of Immunology 1995154 1909-1918.

26 Webster Marketon JI \& Glaser R. Stress hormones and immune function. Cell 2008252 16-26.

27 Biolo G, Iscra F, Toigo G, Ciocchi B, Situlin R, Gullo A \& Guarnieri G. Effects of growth hormone administration on skeletal muscle glutamine metabolism in severely traumatized patients: preliminary report. Clinical Nutrition 199716 89-91.

28 Biolo G, Iscra F, Bosutti A, Toigo G, Ciocchi B, Geatti O, Gullo A \& Guarnieri G. Growth hormone decreases muscle glutamine production and stimulates protein synthesis in hypercatabolic patients. American Journal of Physiology. Endocrinology and Metabolism 2000279 E323-E332.

29 Gelding SV, Taylor NF, Wood PJ, Noonan K, Weaver JU, Wood DF \& Monson JP. The effect of growth hormone replacement therapy on cortisol-cortisone interconversion in hypopituitary adults: evidence for growth hormone modulation of extrarenal 11 betahydroxysteroid dehydrogenase activity. Clinical Endocrinology 199848 153-162.

30 Voerman HJ, Strack van Schijndel RJ, Groeneveld AB, de Boer H, Nauta JP \& Thijs LG. Pulsatile hormone secretion during severe sepsis: accuracy of different blood sampling regimens. Metabolism $199241934-940$.

31 Van den Berghe G, Baxter RC, Weekers F, Wouters P, Bowers CY \& Veldhuis JD. A paradoxical gender dissociation within the growth hormone/insulin-like growth factor I axis during protracted critical illness. Journal of Clinical Endocrinology and Metabolism 200085 183-192.

32 Van den Berghe G, Wouters P, Weekers F, Mohan S, Baxter RC, Veldhuis JD, Bowers CY \& Bouillon R. Reactivation of pituitary hormone release and metabolic improvement by infusion of growth hormone-releasing peptide and thyrotropin-releasing hormone in patients with protracted critical illness. Journal of Clinical Endocrinology and Metabolism 199984 1311-1323.

Received 27 October 2008

Accepted 13 November 2008 